\title{
Variability in X-ray line ratios in helium-like ions of massive stars: the wind-driven case (Corrigendum)
}

\author{
R. Ignace ${ }^{1}$, Z. Damrau ${ }^{1}$, and K. T. Hole ${ }^{2}$ \\ ${ }^{1}$ Department of Physics \& Astronomy, East Tennessee State University, Johnson City, TN 37614, USA \\ e-mail: ignace@etsu.edu \\ 2 Norwich University, 158 Harmon Drive, Northfield, VT 05663, USA
}

A\&A, 625, A86 (2019), https://doi.org/10.1051/0004-6361/201834752

Key words. stars: early-type - stars: massive - stars: mass-loss - stars: winds, outflows - X-rays: stars - errata, addenda

Two equations in Ignace et al. (2019) have minor typos. The first correction is in Eq. (15). That expression has three lines. In the first line, there should be no multiplicative factor of $R_{0}$ appearing. The correct equation is:

$L_{\mathrm{i}}=L_{0} \int\left[\frac{1+2 k_{*} W}{A_{0}-k_{*} \sqrt{1-u^{2}}}\right](1-W) \frac{\mathrm{d} u}{w^{2}}$.

The other two lines in Eq. (15) are correct.

The second correction is in Eq. (19). As in Eq. (15), the appearance of $R_{0}$ is in error. The correct expression is
$L_{\mathrm{i}}=L_{0}^{\prime} \iint\left[\frac{1+2 k_{*} W}{A_{0}-k_{*} \sqrt{1-u^{2}}}\right](1-W) \mathrm{e}^{-\tau(u, \mu)} \frac{\mathrm{d} u}{w^{2}} \mathrm{~d} \mu$.

The mistakes appearing in Eqs. (15) and (19) are typos that have no bearing on the results appearing in the figures which were computed correctly, nor on the discussion or conclusions of the paper.

\section{Reference}

Ignace, R., Damrau, Z., \& Hole, K. T. 2019, A\&A, 625, A86 